Revue de l'Institut des langues et cultures

d'Europe, Amérique, Afrique, Asie et Australie

$38 \mid 2020$

Chant et nation : de la culture populaire à la culture savante

\title{
Rock russe et nation. L'image de la patrie à travers quelques poésies rock des années 1980-2000
}

Russian Rock and Nation. The Image of the Fatherland through some Rock

Poems from the Years 1980-2000

Isabelle Després

\section{OpenEdition}

Journals

Édition électronique

URL : http://journals.openedition.org/ilcea/8225

DOI : 10.4000/ilcea.8225

ISSN : 2101-0609

Éditeur

UGA Éditions/Université Grenoble Alpes

Édition imprimée

ISBN : 978-2-37747-172-0

ISSN : 1639-6073

\section{Référence électronique}

Isabelle Després, «Rock russe et nation. L'image de la patrie à travers quelques poésies rock des années 1980-2000 », ILCEA [En ligne], 38 | 2020, mis en ligne le 31 janvier 2020, consulté le 31 janvier 2020. URL : http://journals.openedition.org/ilcea/8225 ; DOI : 10.4000/ilcea.8225

Ce document a été généré automatiquement le 31 janvier 2020.

(C) ILCEA 


\section{Rock russe et nation. L'image de la patrie à travers quelques poésies rock des années 1980-2000}

Russian Rock and Nation. The Image of the Fatherland through some Rock

Poems from the Years 1980-2000

Isabelle Després

\section{Introduction}

Le film du réalisateur russe Kirill Serebriannikov, Léto, réalisé en 2017 et présenté à Cannes cette année (2018), a permis de mettre le projecteur sur le rock russe, jusque-là largement inconnu en France. Comme le montre le film, la poésie rock en URSS prend un véritable essor au début de la perestroïka gorbatchévienne, de la même façon que le dégel de l'époque de Khrouchtchev avait rendu possible celui de la chanson dite d'auteur ou de bardes, c'est-à-dire la poésie chantée avec accompagnement à la guitare sèche (Blanc, 1991). L'un et l'autre ont bénéficié de la "révolution du magnétophone ${ }^{1}$ ", qui a permis, malgré le contexte de censure et de mainmise de l'État sur la culture, le développement de zones marginales et d'un espace culturel « underground». Si les bardes comme Boulat Okoudjava, Alexandre Galitch, chantent en s'accompagnant d'une simple guitare acoustique, les poètes rock chantent la plupart du temps accompagnés de musiciens (batterie, basse, clavier...) et dans un style rock, c'est-à-dire très rythmé et moins intimiste.

2 Notre propos n'est pas ici de retracer une histoire sociologique complète du rock soviétique, puis russe, ce qui a déjà été fait (Zaytseva, 2008; Zaytseva, 2012), mais de mettre l'accent sur quelques étapes et noms importants, pour enfin nous arrêter, à titre d'illustration, sur l'analyse d'une chanson de Iouri Chevtchouk intitulée «La patrie ». Nous voudrions montrer que, comme tant d'autres emprunts européens (le romantisme, le symbolisme, divers mythes comme celui de Don Juan, etc.), le rock s'est parfaitement acclimaté en Russie. Greffé sur l'arbre de la culture russe, il a donné 
naissance à une branche originale et productive, spécifiquement russe, à la fois dans sa forme et dans son contenu. Il a joué un rôle fédérateur dans le contexte du renversement des valeurs et du chaos qui a suivi la perestroïka. Les textes des chanteurs de rock ont mis des mots sur les doutes et les souffrances des ex-Soviétiques, qui perdaient la patrie tant détestée (parfois), sans que leur en soit proposée une nouvelle.

3 Notre étude ne s'inscrit pas dans l'approche sociologique des «popular music studies ", représentée par Anthony Pecqueux et la revue Volume! (Hein, 2006), mais dans la continuité de nos travaux sur la littérature russe contemporaine. Nous avons volontairement limité l'étude aux trois groupes de rock russe les plus représentatifs. En outre, le choix de notre corpus a été orienté par la présence du thème de la Russie dans les textes.

\section{Un contexte contraignant}

4 Il faut rappeler qu'en URSS, dans le contexte de la guerre froide, la musique dite «rock» en tant que phénomène culturel d'origine occidentale, n'était pratiquement pas diffusée par les canaux officiels, ce qui ne faisait que renforcer son attrait et la fascination qu'elle exerçait sur les jeunes. Pour pouvoir néanmoins exister, les premiers groupes évitaient d'utiliser le mot «rock» et se désignaient comme des «ensembles instrumentaux et vocaux ». Un exemple, parmi les plus connus, est celui d'Andreï Makarevitch et son groupe Machina vremeni (Machine à remonter le temps), qui n'a été officiellement autorisé à donner des concerts sur les scènes professionnelles qu'en 1979, alors qu'il a été fondé en 1969, juste après la fin du dégel ${ }^{2}$.

5 Dans les années 1960 et 1970, comme d'autres mouvements artistiques tels que le conceptualisme (Després, 2012) ou la poésie des bardes, le rock soviétique était confiné strictement à la sphère culturelle alternative, c'est-à-dire non diffusé par les canaux officiels (disques, radio, télévision, concerts), condamné à l'underground et à la clandestinité. Les enregistrements se faisaient non pas en studios, mais dans des appartements privés. Les concerts étaient organisés de façon confidentielle, parfois aussi en appartement ${ }^{3}$, avec un véritable jeu du chat et de la souris entre les musiciens et les auditeurs d'une part, et les organes du pouvoir de l'autre.

6 La poésie rock se développait loin des lieux du pouvoir politique, c'est-à-dire davantage à Léningrad (lieu de pouvoir culturel), ou dans d'autres villes de province, comme Sverdlovsk, qu'à Moscou. Les conditions étaient encore plus favorables en Géorgie, où dès $1980 \mathrm{a}$ eu lieu à Tbilissi le premier festival rock, officiellement intitulé « Rythmes de printemps ». C'est le groupe de Makarevitch qui, cette année-là, a remporté le premier prix. Les autorités renoncent, de fait, à empêcher le développement du rock, et à Léningrad en 1980 se crée le premier «club» de rock, marquant le signe d'une tolérance officielle pour une pratique limitée et canalisée, dans le cadre des activités ou du komsomol. Au même moment, un premier studio d'enregistrement amateur s'organise, sous couvert d'une activité tout à fait officielle d'éveil pour les enfants, le studio d'Andreï Tropillo4. C'est grâce à ce studio semi-clandestin qu'un des premiers compositeurs-auteurs de poésie rock, Boris Grebenchtchikov a pu se faire connaître.

7 Né en 1953, Grebenchtchikov a fondé son groupe Akvarium (Aquarium) en 1971. Le nom du groupe fait allusion à l'existence possible d'un autre monde, au-delà de la paroi, invisible, mais infranchissable, de l'aquarium, métaphore de la vie en URSS. Les 
premiers albums, ceux des années 1970, n'étaient que très peu accessibles, uniquement sur des cassettes ou des disques réalisés avec des moyens rudimentaires et diffusés de façon non commerciale ${ }^{5}$. En 1979, le groupe se fait remarquer au festival de Tbilissi, mais après cela Grebenchtchikov qui travaillait comme mathématicien-statisticien, est renvoyé de son institut de recherche en sociologie, et le groupe est à nouveau victime du harcèlement des autorités. Malgré cela, il continue d'enregistrer et d'organiser des concerts grâce à Andreï Tropillo, et sa notoriété augmente.

Dès le début des années 1980 , le rock russe commence à être différent du rock occidental par la primauté donnée au texte sur la musique. Machina vremeni, Akvarium, avec d'autres groupes de Léningrad ont été les fondateurs du rock à textes. Il faut dire que les moyens techniques sont très limités et que les innovations dans l'accompagnement musical sont encore rares. Les textes d'un autre précurseur, Alexandre Bachlatchev, dont la vie a été courte, puisqu'il s'est suicidé en 1988 âgé de 28 ans, sont aujourd'hui considérés par les spécialistes universitaires russes comme de tout premier ordre (Domanskij, 2010) ${ }^{6}$. Il était arrivé en 1984 de sa province à Léningrad et s'était fondu dans la bohème, fréquentant le club-rock de Léningrad, vivant de petits boulots, de gardien ou de technicien à la fameuse chaufferie Kamtchatka ${ }^{7}$. Ce n'est que dix ans après sa mort qu'il a été reconnu comme un des poètes majeurs du rock russe : en trois ans de cette vie marginale, il a écrit plus de soixante chansons, qu'il interprétait en solo à la guitare acoustique. Ses concerts « en appartement » avaient un grand succès auprès du public de la culture alternative. La poésie de Bachlatchev est marquée par l'influence de Bob Dylan et des bardes russes, en particulier Alexandre Galitch et Vladimir Vyssotski. Il est l'auteur de la chanson «Le temps des clochettes » où s'expriment le malaise et la forte aspiration aux changements d'une génération qui ne croit plus aux idéaux et attend un nouveau dégel, un nouveau printemps. Interprétée pour la première fois en 1984, elle devient, par son énergie électrisante, mais aussi ses images, métaphores et motifs authentiquement russes (la neige, les bouleaux, l'or des coupoles des églises, les clochettes du traineau qui file dans la plaine, le goût enivrant de la vitesse et de l'excès), le symbole et la marque de fabrique du rock russe de ces années 1980 (Kolešev, 2000). Malgré son talent, Bachlatchev n'a pas été reconnu de son vivant et c'est Grebenchtchikov qui a incarné le tournant russe du rock soviétique.

\section{Boris Grebenchtchikov et le tournant russe}

Même si la réputation de Grebenchtchikov dans les milieux rock n'était plus à faire, ce n'est qu'en 1987-1989, grâce à la perestroïka, qu'il devient à la fois célèbre et officiellement reconnu. Il écrit la musique du film Assa ${ }^{8}$ en 1987, et joue dans «Rose noire - tristesse, Rose rouge - amour ${ }^{9} »$ en 1989. Ces deux films étant des événements emblématiques de la perestroïka, ses albums sont dès lors vendus à des millions d'exemplaire, et il passe de plus en plus souvent à la télévision ${ }^{10}$. Mais en 1991, le groupe Akvarium se sépare, Boris Grebenchtchikov continue en solo ${ }^{11}$.

Cette même année, il effectue un périple de cinq mois à travers la Russie, la Sibérie, jusqu'aux îles Solovki. Son style connaît un changement complet, témoignant d'une quête des sources traditionnelles, nationales et populaires. L'album sorti en 1992 s'intitule justement Russkij al'bom (Album russe) et peut être considéré comme une hybridation de musique rock et folk. Sur la pochette du disque vinyle, on voit la figure 
de l'oiseau Sirine, qui renvoie à la chanson de l'album intitulée "Sirine, Alkonost, Gamayun ", du nom des trois "sirènes " de la mythologie russe, mi-oiseaux, mifemmes. L'album contient un grand nombre de motifs religieux orthodoxes, par exemple le titre de la chanson "L'Archi-stratège » (c'est-à-dire l'archange SaintMichel). L'orchestration a recours à des instruments de musique traditionnels populaires, comme la cornemuse russe, la guitare à sept cordes, mais aussi le hautbois et la flûte.

Figure 1. - Pochette de Russkij albom (Album russe) du groupe Akvarium (1991) représentant l'oiseau Sirine.

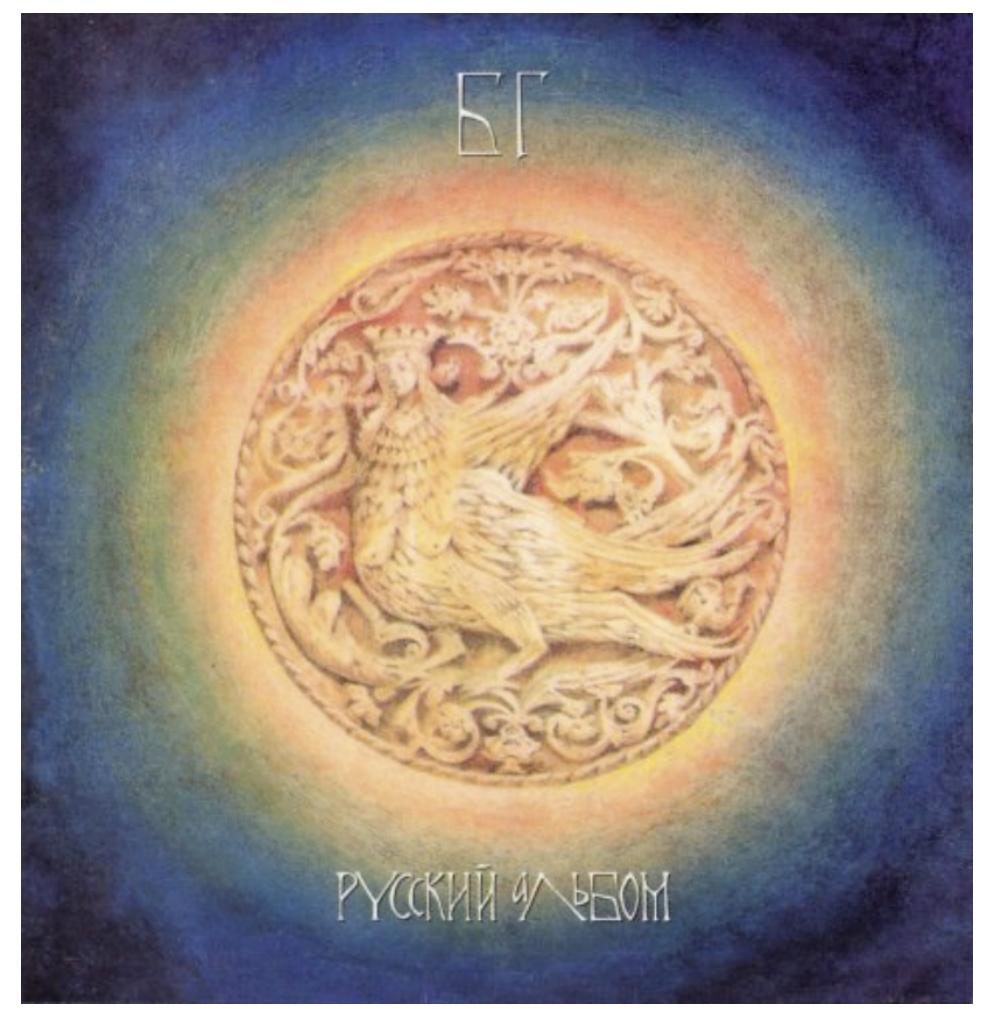

Source : Wikipedia.

Ce tournant se confirme en 1994 avec la sortie de l'album Kostroma mon amour. La recherche d'un son russe passe par les conditions spécifiques de l'enregistrement, qui se fait dans une église (protestante !) de Saint-Pétersbourg. Grebenchtchikov, qui est passionné par le bouddhisme ${ }^{12}$, et semble à cette époque chercher une synthèse avec la partie asiatique de la Russie, puisqu'il invite un groupe de musiciens mongols avec leurs instruments (tambours tibétains, etc.).

12 Le titre de l'album indique cette volonté de synthèse entre ouverture et caractère national, avec l'expression «mon amour » écrite en français et en caractères latins, tandis que Kostroma (nom d'une petite ville typique de la région de la Volga) incarne la province russe. Les paroles sont assez énigmatiques, il semble que le compositeur privilégie les assonances, créant presque une langue inventée à partir des noms de villes: Kostroma / Samara / sestra (sœur) / amour. Les noms des villes russes deviennent comme des incantations magiques. Par la suite, dans l'album « Refuge » (1998), Grebenchtchikov a recours à de véritables mantras tibétains. 

Grebenchtchikov sont riches de symboles empruntés aux mythologies indienne, chinoise, japonaise, celte. Les métaphores sont complexes, obscures, parfois absurdes. Le sens est secondaire, par rapport à la texture. Dans les albums Radio Silence et Radio London, avant 1991, Grebenchtchikov cherche à inventer un système rythmique de la phrase russe qui corresponde à la pulsation de la musique rock. Il expérimente une manière de poser les accents qui rapproche le phrasé d'une métrique purement accentuelle, où l'on ne compte plus le nombre de syllabes, mais uniquement les accents toniques. Il tord parfois la syntaxe russe pour la rapprocher d'une construction de phrase plus anglaise (Agenosov \& Ankudinov, 2006: 422). Il va même plus loin, au tournant des années 1990, en inventant un langage extraterrestre, fait uniquement de sonorités, dans la célèbre chanson "Loï Bykanakh » qu'il interprète dans le film Rose noire - tristesse, Rose rouge - amour ${ }^{13}$.

" chanson "L'ennui vieux russe », dans laquelle Grebenchtchikov a recours à un procédé d'anachronisme, qui consiste à transposer dans la réalité de la Russie postsoviétique les images, les expressions, les locutions figées propres à la tradition culturelle orale ou au patrimoine littéraire classique, ce qui provoque un effet de décalage ironique et désespéré. Il s'agit de motifs issus des récits épiques russes, les bylines, avec leurs personnages semi-historiques, semi-mythologiques (les bogatyrs Ilia Mouromets, Aliocha Popovitch, le prince Vladimir, la lamentation de Iaroslavna), mais aussi des réminiscences littéraires (Les âmes mortes, de Gogol, avec la troïka qui ne peut plus avancer parce que les roues ont été démontées, ainsi que les poèmes populaires de $\mathrm{N}$. $\mathrm{Nekrassov}^{14}$ ). Le sens général est que tout ce qui faisait l'imaginaire russe est mort, couvert de poussière, bon pour le musée. Le refrain est «Je regarde tout cela et je me morfonds d'ennui vieux-russe » (et après le dernier couplet : " J'ai bien peur d'être écœuré par l'ennui vieux-russe »).

Avec son constat de la mort de la culture et sa désillusion, Grebenchtchikov se situe dans le paradigme du postmodernisme (Després, 2008a), mais ses expérimentations linguistiques et son attirance pour la philosophie orientale et le bouddhisme sont caractéristiques du postmodernisme russe (Lipoveckij, 1997 ; Després, 2008b ; Epstein \& Genis, 2016), dans la mesure où pour lui le mot reste sacré, il conserve sa force incantatoire, et la désillusion laisse finalement place à la recherche d'une nouvelle spiritualité.

\section{Viktor Tsoï et la naissance d'un mythe}

Boris Grebenchtchikov a ouvert la voie d'un rock russe, mais le personnage le plus emblématique des années de la perestroïka restera Viktor Tsoï, le chanteur du groupe Kino, que sa mort précoce a élevé au statut d'idole des jeunes et de véritable légende. Né en 1962, il appartient à une autre génération que celle de Grebenchtchikov. En 1981, il fonde avec des amis un groupe qui deviendra Kino en 1982. Le film de Kirill Serebriannikov Léto retrace l'été 1981 de V. Tsoï à Léningrad et la fondation du groupe Kino $^{15}$. Les jeunes rockers enregistrent dans le studio d'Andreï Tropillo, avec l'aide des musiciens de Grebenchtchikov. Mais comparé au rock de Grebenchtchikov, aux accents de musique populaire, le son de Kino est bien plus électrique, voire métallique, avec un recours à l'électronique, aux synthétiseurs. Commencent des concerts en appartement à Léningrad et à Moscou. Viktor Tsoï écrit des chansons emblématiques, comme « Les 
cornichons d'aluminium » [АлюмИнЕвыЕ огурцы] ou «Je déclare ma maison zone antinucléaire » [я ОБъЯВляю дОм Свой БЕзъЯдЕРной зоной], manifeste pacifiste contre la guerre et contre la bombe atomique. Il est de plus en plus populaire, tout semble lui sourire, puisqu'il se marie et a un fils en 1985. En 1986 sort l'album «La nuit » [ночь] dans lequel on découvre encore plusieurs morceaux significatifs, comme « On a vu la nuit » [ВИДЕЛИ НОчь] et « Ma mère, l'anarchie » [МАмА - АНАРХИЯ ].

17 Tout en faisant des ménages de nuit ou en travaillant comme chauffagiste (à la chaufferie Kamtchatka) Viktor Tsoï donne des concerts semi-clandestins dans de nombreuses villes, où il est invité. En 1987, un film documentaire du célèbre réalisateur Alekseï Outchitel intitulé Rock ${ }^{16}$ provoque un certain scandale. Il raconte, et d'une certaine façon, donne une existence à ce phénomène social qui est toujours non reconnu par les médias officiels d'État. Le film montre le malaise d'une jeunesse qui n'en peut plus, qui étouffe. Le rock exprime aussi un conflit de génération du type pères/fils, car souvent les adultes ne comprennent pas ces sons sauvages, cette violence, cette façon de s'habiller provocante (maquillage noir, strass, tatouages).

Viktor Tsoï compose la chanson «Des changements " pour le festival de rock de 1986, c'est-à-dire avant l'introduction par Gorbatchev des véritables changements. La chanson devient un tube des années de la perestroïka, elle est incluse dans la scène finale du film culte Assa. Elle fera ensuite partie de l'album «Le dernier des héros » (1989). Dans le contexte des bouleversements socio-politiques qui s'ensuivent, elle est adoptée et reprise en chœur par le public comme un hymne. Jusqu'à nos jours, la chanson est un signe de ralliement des contestataires ${ }^{17}$.

Figure 2. - Pochette de l'album Poslednij geroj (Le dernier des héros) du groupe DDT (1989).

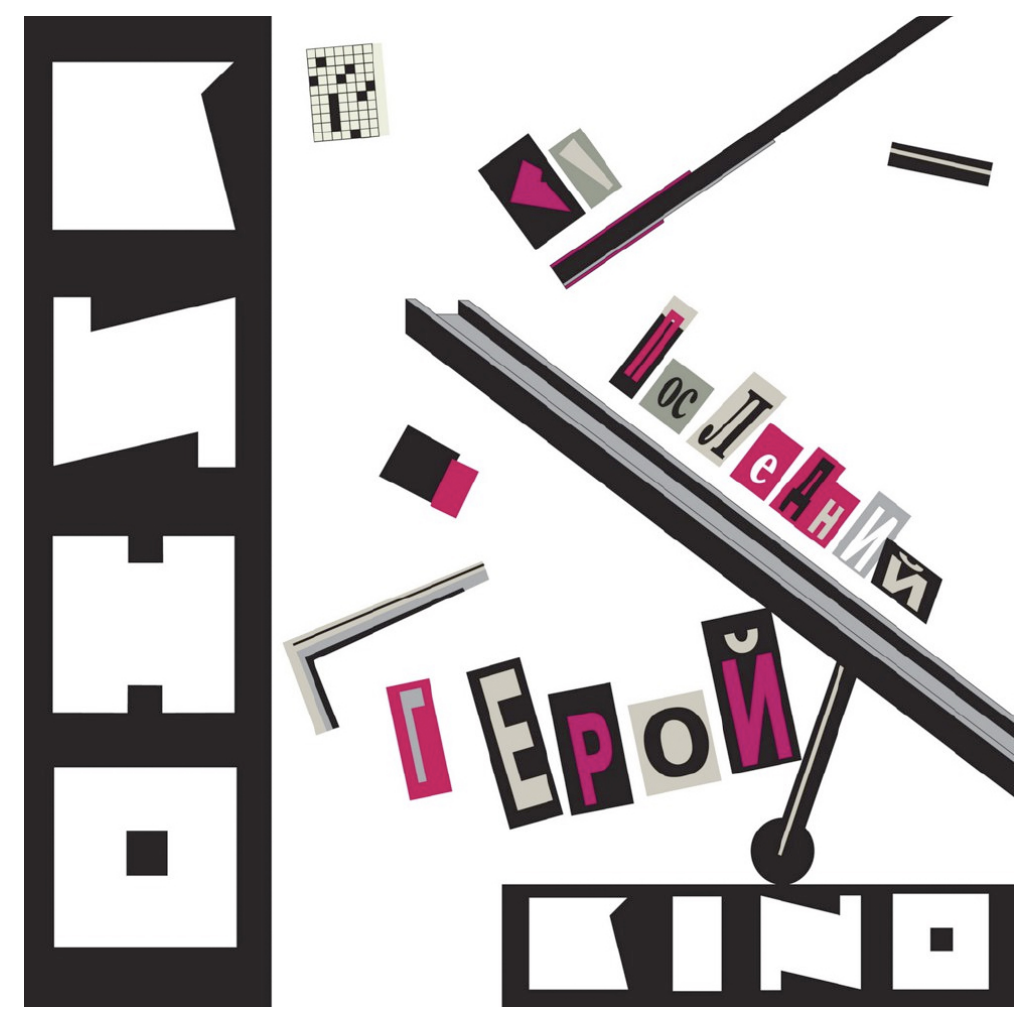

Source : Wikipedia. 

ce groupe, qui deviendra DDT, sort bientôt un premier album au style allant du hard rock au rythm and blues, avec dès le début des accents folk, et beaucoup d'éléments rappelant la chanson populaire et le folklore russe. Il faut rappeler qu'en 1980, surtout au Bachkortostan, on commence à entrevoir la réalité de la guerre en Afghanistan, malgré la propagande officielle, qui parle de développement et d'amélioration du niveau de vie des Afghans, et cela d'autant plus que les premiers cercueils de soldats russes arrivent à Oufa. Chevtchouk écrit une chanson qui deviendra un slogan pacifiste: «Ne tire pas!»[НЕ СТРЕЛЯЙ] Avec cette chanson, le groupe 
remporte inopinément le premier prix du festival musical d'Oufa en 1982, et acquiert une certaine notoriété.

Mais les autorités harcèlent Chevtchouk. La presse le traite "d'agent du Vatican » (pour une chanson sur Jésus, intitulée "Nous emplirons le monde de bonté»), les autorités du Bachkorstan ne peuvent lui pardonner d'avoir représenté leur pays comme une " périphérie ». On lui reproche de donner une image peu rieuse du pays. Il est exclu du Komsomol, mais il continue à faire des concerts privés en appartement, puis il s'installe à Léningrad, où il mène la vie d'artiste de bohème, travaille comme gardien de nuit, chauffagiste, tout en se consacrant à la musique.

À la faveur de la perestroïka (1987), il rassemble un nouveau groupe de musiciens, le «DDT de Léningrad ». Un premier succès incite la firme monopoliste officielle Melodia à produire un album de DDT qui s'intitule «J'ai reçu ce rôle » [я получил эту Роль]. Alors commence l'heure de gloire, couronnée par un grand concert en plein air, le 20 mars 1993 sur la place du Palais d'Hiver, à Saint-Pétersbourg, où sont rassemblés plus de 120000 personnes. Entre 1990 et 1999, DDT enregistre sept albums, dont le plus significatif est en 1992 "L'actrice Printemps» [АКТРИСА ВЕСНА], avec la chanson «Automne » [OCEHb] qui a été couronnée par un record d'audience à la radio.

Par la suite, Chevtchouk demeure une célébrité. Toujours méfiant envers le pouvoir, il s'engage avec constance contre les guerres. En 1999, pour protester contre les bombardements de l'OTAN en Serbie et au Kosovo, il fera un documentaire sur la destruction des églises orthodoxes du Kosovo. Puis il proteste contre la guerre russe en Tchétchénie, où il se rendra pour donner quatre concerts, dont trois en 1995, quasiment sous les balles, et un après la paix. Il se brouille avec le groupe Kino, auquel il reproche d'avoir cherché à gagner de l'argent grâce au disque posthume confectionné après la mort de Viktor Tsoï, renforçant ainsi son image de chanteur éthique, pacifiste, patriote sans être chauvin, intransigeant avec le pouvoir, en particulier dans les années 2000 et 2010.

29 En 2000, Chevtchouk exprime l'émotion populaire ressentie lors de la tragédie du sousmarin Koursk par une chanson bouleversante, «Le Capitaine Kolesnikov », dans laquelle il accuse explicitement les autorités de ne pas dire la véritée ${ }^{1}$. Il est du côté des révolutionnaires à Kiev lors de la révolution orange (2004-2005). En 2007 il participe à la Marche du désaccord à Saint-Pétersbourg ${ }^{22}$. En 2014, il condamne l'intervention de troupes russes en Ukraine, mais appelle les deux côtés à la paix, et envoie de l'argent pour soutenir les habitants du Donbass pro-russe. Il ne cesse de dénoncer comme une censure le fait que certaines de ses chansons ne soient jamais diffusées à la radio. Lors d'une rencontre organisée avec les représentants du monde de l'art ${ }^{23}$, Poutine, sentant venir ses questions gênantes, essaie de le déstabiliser, en lui demandant, en dépit de sa popularité : «Pardon, vous êtes qui, vous? », et cette phrase reprise en boucle devient un mème internet.

Amené à s'intéresser à la religion orthodoxe dès les années 1970 par soif de spiritualité et esprit contestataire, Chevtchouk aujourd'hui encore s'affirme croyant, de culture religieuse et orthodoxe, tout en se méfiant fondamentalement de l'Église et en condamnant le rôle politique que se donne le Patriarche russe, qui appelle à voter pour Poutine. Mais il ne refuse pas de participer à des concerts religieux et même de chanter les vers écrits par un prêtre orthodoxe, comme ceux de l'album « Maintenant, Seigneur, tu laisses ton serviteur » [НЫНЕ ОТПУЩАЕши] dont le titre est une citation d'un texte liturgique. 
31 Sa position est profondément morale : il faut commencer par changer soi-même avant de changer le monde. Il ne suffit pas de désigner Poutine comme responsable de tous les maux de la Russie, car c'est à la société russe elle-même de se débarrasser des restes du soviétisme. Chevtchouk est intransigeant pour la culture dite " pop », c'est-à-dire la chanson facile pour les masses et les chanteurs comme Kirkorov, auxquels il dénie toute recherche spirituelle. Le consumérisme, avec son corollaire, l'argent, lui apparaît comme une dérive dont il faut coûte que coûte se préserver. Cette position fait de Chevtchouk une autorité de référence pour de nombreux Russes. La ferveur et l'émotion qu'il suscite lors de ses concerts en sont le signe.

Chevtchouk propose dans ses chansons un nouveau rapport à la Russie en tant que nation. Ici, il semble utile de préciser la sémantique des trois mots russes qui peuvent signifier «nation » ou "patrie ». Deux d'entre eux sont formés sur la racine du mot " père » (comme " patrie » en français), l'un est neutre : otechestvo, l'autre est féminin (comme en français), c'est otchizna. Mais il existe aussi le mot rodina qui est étymologiquement plus proche de "nation ", puisque c'est la racine de natio, naître, soit en russe rodit', qui est à l'origine de ce mot (à entendre donc comme terre «natale »). Enfin, il existe le mot russe d'origine latine, natsia, qui est peut-être plus proche du sens du mot français "nation ", mais qui, étant senti comme étranger à la langue russe, ne porte aucune connotation affective. Il existe aussi le mot narod, qu'on traduit habituellement par "peuple » alors qu'il est bien plus proche de "nation ", puisqu'il est aussi construit sur la racine de rod, c'est-à-dire le lien de naissance, de filiation, et qu'il introduit l'idée de famille. Or, on ne renie pas sa famille, même lorsqu'adolescent on voudrait, peut-être, en avoir une autre. C'est ce sentiment d'attachement, d'amour inexpugnable qu'on peut avoir pour une vieille mère dont on a un peu honte, que chante Chevtchouk, en particulier dans la chanson Rodina ${ }^{24}$.

Dieu, Tant d'années que je marche

Et je n'ai pas fait un pas

Dieu, Tant d'années que je cherche

Ce qui est éternellement en moi

Tant d'années que je mâche non du pain,

Mais mon amour indigeste

Tant d'année que me crache à la nuque le canon noir-corbeau

Des lointains attendus

Les phares noirs au porche voisin

Trappes, menottes, ma bouche écorchée

Combien de fois ma tête a roulé du billot

Trop plein, pour atterrir ici, où est

Ma Patrie ! Je reviens dans ma Patrie !

On peut crier qu'elle est affreuse

Mais elle nous plaît, oui elle nous plaît.

Elle n'est pas belle, elle fait confiance aux salauds,

Mais nous on s'en... tra la la

Dieu! Que de vérité dans les yeux des putains de l'État

Dieu! Que de foi dans les mains des bourreaux retraités

Ne les laisse pas retrousser leurs manches à nouveau,

Leurs manches de nuits blanches affairées

Les phares noirs au porche voisin

Trappes, menottes, ma bouche écorchée

Combien de fois ma tête a roulé du billot

Trop plein pour atterrir ici où est 
Ma patrie! Je reviens dans ma patrie !

On peut crier qu'elle est affreuse

Mais elle nous plaît, oui elle nous plaît.

Elle n'est pas belle, elle fait confiance aux salauds,

Mais nous on s'en... tra la la

Sous les chemises noires le coq rouge veut bondir

Sous les bons rois on vous verse dans la bouche de la confiture

Jamais ce monde n'a pu contenir les deux

Dieu était notre père, le diable notre

Mère patrie! Je reviens dans ma patrie!

On peut crier qu'elle est affreuse

Mais elle nous plaît, oui elle nous plaît.

Elle n'est pas belle, elle fait confiance aux salauds,

Mais nous on s'en... tra la la

Oh là, chef !

Cette chanson a été immédiatement reprise dans l'album intitulé "L'actrice Printemps » en 1992. Or, le regroupement des titres en albums a autant d'importance pour le rock que, pour la poésie, la constitution de recueils de poèmes. Le visuel et le titre de l'album sont également significatifs.

Figure 3. - Pochette de l'album Aktrisa Vesna (L'actrice Printemps) du groupe Kino (1992).

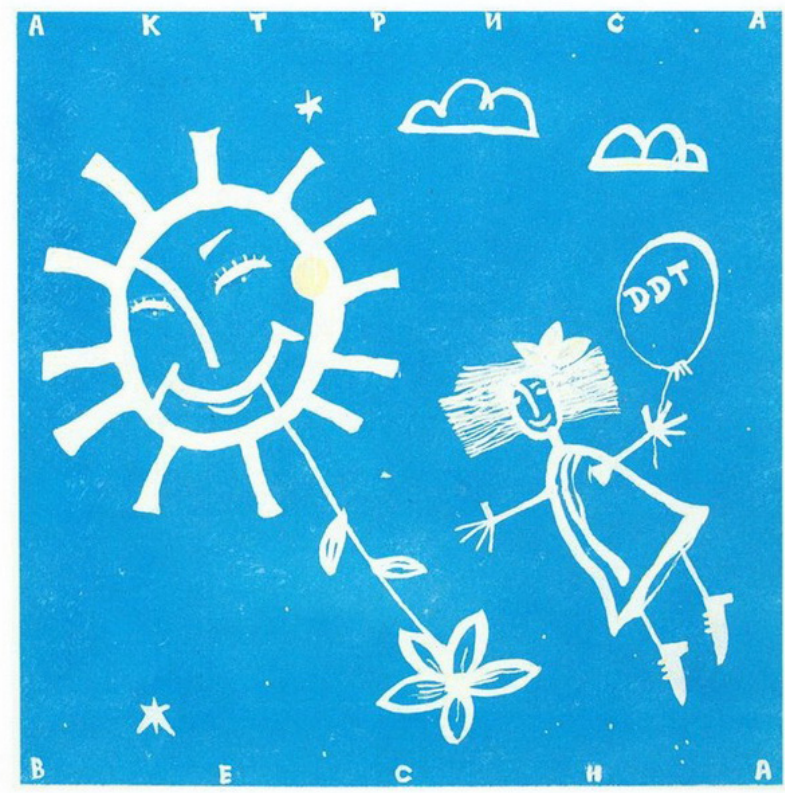

Source : Wikipedia.

Le titre de cet album de Chevtchouk fait à nouveau référence au motif du dégel (un précédent album en 1990 s'intitulait déjà « Dégel »), c'est-à-dire au renouveau de la nature et de la vie (le "printemps »). Ce thème est renforcé par le dessin d'enfant qui illustre la couverture de l'album. Mais par ailleurs, le mot « actrice» fait référence au jeu de l'acteur, qui est proche du « jeu » postmoderniste, ou encore à l'illusion que sait provoquer l'actrice, la femme charmeuse. C'est ainsi que s'incarne la féminité dans 
l'œuvre du grand poète symboliste du début du $\mathrm{xx}^{\mathrm{e}}$ siècle, Alexandre Blok, qui, comme tant d'autres, avait une véritable passion pour les actrices. Dans le célèbre poème "L'inconnue ${ }^{25}$ " [НЕзНАКомКA] il esquisse cette femme séduisante, mais froide et hautaine, ambiguë et ambivalente, à la fois ange et démon, qu'il peindra dans le cycle "Carmen » sous les traits d'une actrice, jouant avec le cœur de son admirateur comme Carmen avec Don José. Plus tard, dans le recueil intitulé « Vers sur la Russie » et dans le poème sur la révolution, "Les Douze ", la féminité dans l'œuvre de Blok tend à se confondre avec la Russie.

Justement, cette séduisante actrice inconnue de l'album de Chevtchouk, ce pourrait être l'époque, mais c'est surtout la patrie, qui est capable du meilleur, comme du pire. L'amour du chanteur-poète pour la Russie est aussi « indigeste » que celui de Blok pour la Russie révolutionnaire.

La chanson Rodina résonne, selon le statut que l'on donne à l'interjection " Dieu! », au premier vers, comme un cri ou une prière. La prière l'emporte, certes, au deuxième couplet, avec la forme du verbe à l'impératif, dans « Ne les laisse pas retrousser leurs manches à nouveau ».

Finalement, la voix du chanteur, l'interprétation, ne laissent pas de place au doute, c'est une lamentation. Le poète est dans un temps sans début ni fin, où rien ne change : les verbes sont au présent, ou à un passé de réitération. C'est une répétition tragique, une mise à mort sans cesse renouvelée, une exécution capitale en place publique. Le poète, pourtant, même si c'est après sa mort, "revient dans sa patrie ", qui apparaît, par conséquent, comme un au-delà d'outre-tombe. Sa tête $\mathrm{y}$ "roule » après avoir été coupée.

38 La patrie est loin d'être un pays idéal. D'ailleurs le pays idéal (le «lointain attendu ", c'est-à-dire une utopie équivalente à « l'avenir radieux » du communisme) se trouve paradoxalement derrière le poète, derrière sa "nuque ", sur laquelle il pointe "un canon de fusil » («noir » et froid, comme la mort, symbolisée par le «corbeau »). Les espoirs se sont brisés contre les répressions, les arrestations nocturnes ("phares noirs »), les tortures (« trappes », « menottes », etc.). Mais le refrain l'affirme : la patrie, même dénaturée, désacralisée, est encore aimée.

Il faut remarquer le passage du "je » lyrique au «nous » (" elle nous plaît»), le poète endossant là le rôle de porte-parole épique. On retrouve à maintes reprises ce passage $\mathrm{du}$ «je » au «nous» dans les chansons de Chevtchouk, voire l'opposition «eux» / «nous ", révélant généralement une conception binaire du monde où la Russie s'exclut de l'Europe et de l'Occident. Toutefois, cette opposition est mouvante chez Chevtchouk. Par exemple, dans la chanson «En cette ... » [В это...], le «nous» et le «vous» semblent interchangeables ${ }^{26}$ :

Ici pas d'autre mouvement que les bandes criminelles, l'eau bénite ne protège pas de la mort

On nous a autorisés à nous lever, mais on n'a nulle part où aller : personne ne sait pourquoi faire et où.

Ici le ciel est fait de discorde, et la terre, de problèmes, le corps exige du savon, et les âmes, une chaudière

Et nous voulons croire, mais nous ne savons pas en quoi,

Ici pas de moisson, depuis six ans c'est le printemps

Ici on arrache les vaches, on a des infirmes au déjeuner, des orphelins pour le dîner, $\mathrm{y}$ chantent des sottises des messieurs au sex-appeal, et l'avenir poisseux à nouveau nous écrasera, pauvres pêcheurs, avec son jeûne gras 
ce mensonge total sur ce que sera demain, des sages nous disent qu'il est bon pour nous

Nous sommes si habitués à la merde que l'appel à la propreté ne vous nettoie pas les oreilles, ne nous décille pas les yeux

Temps des jeunes lois, des nouveaux embryons, mais la mortalité infantile, hélas, est élevée

Et le temps qui n'est pas né a déjà l'odeur de la malédiction, comme notre mer et votre rivière

En cette nuit tu ne remarques que deux couleurs d'yeux, la peur se fige chez les intelligents comme la colère chez les imbéciles

Ici l'activité des foules est sens dessus dessous, la jeunesse est sage, mais la vieillesse cruelle

Quand on ne sait plus où ramper, on sait encore où s'enfuir, après l'incendie on vous y réchauffera le cœur

Mais là-bas je n'ai pas de quoi vivre et pas de raison de mentir, ici j'ai ma mère, et je crois dans le Christ

ici repose mon frère et je regarde pousser les bouleaux sur sa poitrine

Que pourrissent les ennemis, que trahissent les amis, je continue à vivre, je continue à avancer vers cette - haha - sublime époque. Reviakine, malgré leurs différences, conforterait notre thèse qu'après un réel engouement pour la musique de l'Occident, s'est opéré un mouvement de retour vers la thématique de la terre natale, de la Russie, et vers des sonorités plus résolument nationales et populaires.

Ainsi, la poésie rock est un phénomène représentatif de la culture russe, en tant qu'appropriation d'un modèle étranger (ici, le rock à la manière de Bob Dylan) par hybridation avec les modèles russes, comme la poésie d'Alexandre Blok et de l'âge d'argent, ou la chanson d'auteur (en particulier celle de Vladimir Vyssotski, dont la voix rauque et les textes percutants sont proches de la poésie rock), même la poésie populaire comme la chanson des camps ${ }^{31}$. 


\section{BIBLIOGRAPHIE}

AGENOSOV Vladimir V. \& ANKUDINOV Kirill N. (dir.) (2006), « Rok poeziâ », Sovremennye russkie poety: Antologiâ, Moscou : Verbum, 421-461.

BLANC Hélène (1991), Les auteurs du Printemps russe : Okoudjava, Galitch, Vyssotski, Montricher : Les éditions noirs sur blanc.

DESPRÉS Isabelle (2008a), « Modernisme et post-modernisme : une révision des valeurs de l'âge d'argent à l'époque de la post-modernité ? ", Modernités russes, 7, 659-670.

DESPRÉS Isabelle (2008b), « La réception et la transposition des concepts occidentaux en Russie. Postmodernisme et post-modernité ", S. Martin (dir.), Circulation des concepts entre Occident et Russie, Lyon : ENS LSH, <http://institut-est-ouest.ens-lsh.fr/spip.php?article> (8 mai 2019). DESPRÉS Isabelle (2012), « Le conceptualisme moscovite : art progressiste ou dissidence idéologique ? », ILCEA, 16, <http://dx.doi.org/10.4000/ilcea.1351>. 
DoMANSKIJ Ûrij V. (2010), Russkaâ rok poeziâ: Tekst i kontekst, Moscou : Intrada, Izd. Kulaginoj.

KoŠELEV Vâčeslav Anatol'evič (2000), « “Vremâ kolokol'cikov”: literaturnaâ istoriâ simvola », Russkaâ rok poeziâ: Tekst i kontekst, 3.

EPSTEIN Mikhail, GenIS Alexander A. \& VLADIV-GLover Slobodanka M. (2016), Russian Postmodernism: New Perspectives on Post-Soviet Culture, New York : Berghahn Books.

HEIN Fabien (2006), « Le Monde du rock », Volume !, <http://journals.openedition.org/volume/ 2611> (8 mai 2019).

LEMAIRE Frans C. (2005), Le destin russe et la musique. Un siècle d'histoire de la Révolution à nos jours, Paris : Fayard.

LIPOVECKIJ Mark N. (1997), Russkij postmodernizm, Ekaterinbourg, Russie : Université de l'Oural.

MARKelova Olga A. (2000), «Tema rodiny v poezii Û. Ševčuka », Russkaâ rok poeziâ: Tekst i kontekst, $3,19-26$.

ŠADURSKIJ Vladimir V. (2014), « Ohota na volkov: pesni V. S. Vysockogo u istokov russkogo roka », Russkaâ rok poeziâ: Tekst i kontekst, 15, 69-77.

ZAYTSEVA Anna (2008), “ “À la scène comme à la ville” : engagements multiples des musiciens underground », Ethnologie française, 38(1), 129-137, <https://halshs.archives-ouvertes.fr/ halshs-00695661>.

ZAYTSEVA Anna (2012), En quête d'Altérité : pour une sociologie des acteurs, lieux et pratiques de la scène rock à Léningrad/Saint-Pétersbourg dans les années 1970-2000 (thèse de doctorat), EHESS, Paris, $<$ www.theses.fr/2012EHES0019>.

Les textes en russe sont accessibles sur <www.gl5.ru>.

Pour Boris Grebenchtchikov : <www.gl5.ru/aquarium.html>.

Pour Viktor Tsoï : <www.gl5.ru/coi-viktor.html>.

Pour Iouri Chevtchouk : <www.gl5.ru/ddt.html>.

\section{NOTES}

1. Prenant, d'une certaine façon, la relève du samizdat, le magnitizdat consiste à diffuser la culture underground grâce au magnétophone à cassettes, dont l'usage se répand, ce qui permet d'enregistrer des récitals ou concerts privés ou interdits.

2. Né en 1953, le jeune Makarevitch était un fan des Beatles. Avec son lyrisme philosophique, il incarne mieux que quiconque le lien de continuité entre la chanson d'auteur et le rock, d'autant plus qu'il chante aussi en solo. Il a été exclu de l'Institut d'architecture, où il faisait ses études, et pendant dix ans, il a subi le harcèlement des autorités.

3. Voir la longue séquence vers la fin du film Léto de Kirill Serebriannikov. L'entrée était le plus souvent payante. Aujourd'hui, cette ambiance intimiste et familiale est recréée artificiellement dans la mise en scène des concerts de Iouri Chevtchouk pour leur retransmission télévisuelle, ce qui traduit, peut-être, une forme de nostalgie, assez répandue par ailleurs.

4. Andreï Tropillo était l'animateur officiel d'un club d'initiation aux techniques audiovisuelles, pour les enfants, au palais des Pionniers de Léningrad. Le soir, après les activités périscolaires, il mettait clandestinement le studio d'enregistrement à la disposition des jeunes musiciens rock amateurs. 
5. Certains concerts pouvaient être gravés sur vinyle, mais ces enregistrements étaient de mauvaise qualité et confidentiels. On pouvait aussi se procurer des disques gravés sur d'anciennes radiographies médicales, d'où le terme évocateur de « rock sur les os » employé pour désigner ce mode de diffusion.

6. La « rockologie » est aujourd'hui en Russie un domaine d'étude reconnu, avec des spécialistes tels que le professeur Iouri Domanski, ainsi que des publications scientifiques régulières (telles que la revue scientifique Texte et contexte, qui paraît régulièrement depuis 1998).

7. Les artistes bohèmes se passaient le filon : être chauffagiste de nuit à la chaufferie Kamtchatka (en plein centre de Léningrad) permettait de vivre à l'écart du système, en marginal, et de se sentir libre. Le travail consistait à verser du charbon dans la chaudière. On y organisait aussi des concerts clandestins de rock. Le film Rock de 1987 y a été tourné. Aujourd'hui le bâtiment est devenu le musée Viktor Tsoï.

8. Film culte du réalisateur Sergueï Soloviov, auquel participe également, en tant qu'acteur, le chanteur Viktor Tsoï.

9. ЧЁРНАЯ РОЗА - ЭМБЛЕМА ПЕЧАЛИ, КРАСНАЯ РОЗА - ЭМБЛЕМА ЛЮБВИ, film du même Sergueï Soloviov dans lequel Grebenchtchikov joue en tant qu'acteur. La musique est interprétée par le groupe Akvarium.

10. Dans l'émission de divertissement Vesëlye rebâta (Les joyeux garçons) qui a existé de 1979 à 1990.

11. Mais par la suite il fonde Akvarium 2 qui entre 1992 et 1997 sort 7 albums. Akvarium 3 est créé en 1999 et existera jusqu'en 2013, ce qui correspond aux années Poutine. Signe de son officialisation, Grebenchtchikov fête ses 50 ans par un grand concert au Kremlin.

12. La passion de Grebenchtchikov pour le bouddhisme est en partie inspirée par George Harrison. Il est intéressant, pour notre propos, de souligner le détour par l'Occident, via les Beatles, pour aboutir à cet intérêt pour l'Orient spirituel, qui n'est pas seulement le fait des musiciens de rock, mais aussi des écrivains comme Viktor Pélévine (voir son roman La mitrailleuse d'argile) et autres artistes et acteurs de la vie culturelle.

13. Cette expérimentation ne manque pas de rappeler le langage transmental que recherchaient les futuristes russes, en particulier V. Khlebnikov. Voici les étranges paroles de cette chanson : « БЫШЛИ ХЛАЙ, БЫШЛИ ХЛАЙ, БЫШЛИ ХЛАЙ, ЛОЙ БЫКАНАХ ».

14. Poète populiste du $\mathrm{XIX}^{\mathrm{e}}$ siècle dont les vers sont connus de tout écolier russe.

15. Grebenchtchikov a critiqué le scénario de ce film, et les personnages, qui d'après lui ne ressemblent pas à leurs modèles. Serebriannikov en aurait fait des « hipsters moscovites ».

16. Le film révèle au large public l'existence des groupes de rock dont il est question dans cet article, Akvarium (Grebenchtchikov), Kino (Tsoï) et DDT (Chevtchouk), et d'autres. Certaines scènes sont tournées dans la chaufferie Kamtchatka.

17. Par exemple, on prétend qu'elle est interdite à la radio biélorusse (source wikipedia).

18. Т. Aitmatov, Les rêves de la louve [ПЛАХА], trad. C. Zeytounian, Messidor, 1987.

19. "J'ai une tête de Tatare avec un nom d'Ukrainien " [ТАТАРИН НА лИЦО ДА С ФАМИЛИЕЙ хохляцкой], dit Chevtchouk de lui-même dans une chanson.

20. Les deux grands-pères de Chevtchouk ont été fusillés dans les années 1930.

21. «Qui nous dira sur leur mort un ou deux mots de vérité

Dommage que les marins tombés n'aient pas de boîtes noires [...]

Après, on va longtemps nous mentir sur ce qui s'est passé » (traduit et souligné par I. Després).

22. Manifestation de protestation contre la falsification des résultats des élections.

23. Cette rencontre a eu lieu à Saint-Pétersbourg, au théâtre Mikhailovski fin mai 2010.

24. Nous proposons ici notre propre traduction des vers de Chevtchouk.

25. "L'Inconnue", traduction du poème suivie de trois articles, "Traduire l'Inconnue, Inactualité de Blok et De l'obscur», dans Éclats d'une poétique du devenir posthumain par Serge Venturini, Livre II, Paris, Éditions L’Harmattan, coll. « Poètes des cinq continents », 2007. 
26. C'est nous qui traduisons et qui soulignons (I. Després).

27. Voir aussi la chanson « Le ciel sur terre » [НЕБО нА ЗЕмлЕ] avec le poète saoul et l'ange ivre [ ХмЕЛЬНОЙ ПОэт ПьянЫЙ АНГЕЛ].

28. «J'aime ma patrie, mais d'un amour étrange!» [люБлю отчизну я, но стРАнною люьовью!], dit le poète romantique russe dans un poème de 1841 intitulé Rodina.

29. Voir la chanson « Interview » [ИНТЕРВью].

30. [стРАшный мИР] Le Monde terrible, traduit du russe et présenté par Pierre Léon, Poésie/ Gallimard, 2003.

31. Chanson des bagnards, des camps de travail soviétiques [БЛАТНАЯ ПЕСНЯ].

32. Voir «Dernier automne » [ПослЕДняя осЕнь] (Chevtchouk) pour Pouchkine. Et «Pluie » [ дождь] (Chevtchouk) pour Tioutchev et son poème « Orage au début du mois de mai » [люБлю ГРОЗУ В НАЧАЛЕ МАЯ].

\section{RÉSUMÉS}

L'article s'intéresse au rock russe des années ayant précédé et suivi la perestroïka. Après un rappel $\mathrm{du}$ contexte, il met en évidence une évolution vers les procédés de la musique traditionnelle et vers une poétique du sentiment d'appartenance nationale à la Russie postsoviétique. Il montre qu'en renouant avec le motif tragique de l'amour-haine pour la patrie et de la souffrance d'être russe, le rock russe s'affranchit de ses modèles occidentaux et s'inscrit dans la tradition littéraire russe. Pour cela il étudie les paroles de plusieurs chansons des chanteurs Grebenchtchikov, Tsoï et Chevtchouk, ainsi que leurs groupes respectifs, Akvarium, Kino et DDT.

The article focuses on Russian rock from the years before and after perestroika. After a reminder of the context, it highlights an evolution towards the processes of traditional music and towards a poetics of national attachment to post-Soviet Russia. By reviving the tragic motive of love-hate for the country and the suffering of being Russian, Russian rock is freeing itself from its Western models and becomes part of the Russian literary tradition. To prove that, the article studies the lyrics of several songs wrotten by the singers Grebenchchikov, Zoï and Shevchouk, as well as their groups, Akvarium, Kino and DDT.

\section{INDEX}

Keywords : Post-Soviet Russia, perestroika, Russian rock, rock poetry, homeland, national sentiment, Grebenchchikov, Zoï, Shevchouk

Mots-clés : Russie post-soviétique, perestroïka, rock russe, poésie rock, patrie, sentiment national, Grebenchtchikov, Tsoï, Chevtchouk 
AUTEUR

\section{ISABELLE DESPRÉS}

ILCEA4, Université Grenoble Alpes

isabelle.despres@univ-grenoble-alpes.fr 